\title{
Sociología y modernidad: cómplices de una época anunciada
}

\author{
Martuccelli, Danilo (1999), Sociologies de la modernité, \\ Éditions Gallimard, Col. Folio essais/Inédit, París, 709 PP., \\ ISBN: 2-07-041050-1
}

Si la Modernidad es reacia a toda definición exhaustiva, es que su utilidad analitica proviene justamente de su indecisión conceptual, de su capacidad de dar cuenta de un gran numero de fenómenos dispersos en bastantes disciplinas, así como de un número no menos considerable de polémicas.

Danilo Martuccelli.

Al hablar de Sociologías de la modernidad diríamos que se trata de un pleonasmo, sobre todo cuando se refiere al título de un libro. Quizá esto es lo que atrae a primera vista de la propuesta de Danilo Martuccelli. Suele suceder con frecuencia que cuando algo es evidente, se tienden a olvidar o al menos a perder de vista las relaciones de origen, particularmente cuando se trata de la relación estricta que mantiene un término, noción o concepto con su descriptor, o, para el caso, se tiende a olvidar el lazo existente entre formas de abarcar y comprender las relaciones humanas (sociología) y el espíritu del tiempo que las sustenta (modernidad). Igual sucede con los análisis derivados de los llamados empirismos lógicos que se empeñan en buscar las evidencias que fundamenten las teorías de origen, más que abocarse a describir y comprender lo que es evidente, y no forzosamente visible, en la dinámica de lo social.

Lo atractivo y a la vez complejo que se encierra en esta relación (sociologías-modernidad), inscrita desde los primeros capítulos del mismo libro, no está en el reconocimiento de que la sociología está inexorablemente vinculada con la modernidad, sino en el cuestionamiento que recorre todo el análisis del texto; a saber: si habría sociologías sin modernidad, o si la modernidad hubiera podido advenir sin el nacimiento de la misma sociología. ¿Quién pudiera negar que las obras de un tal Maquiavelo, las deliberaciones inertes en unas Cartas persas o la evocación del aforismo "cogito ergo sum" (pienso, luego existo) -todo ello precursor del pensamiento sociológico- serían testimonio y alimento de la fundamentación misma del proyecto y pensamiento de la modernidad? Más aún, no falta quien sugiera que con el estable- 
cimiento del platonicismo-cristiano en los siglos IV y vi a.C., el proyecto inscrito en la modernidad encontraría más tarde las raíces de su emancipación, a partir del siglo XVI, y por tanto la necesidad de conformación en un segundo plano de herramientas de análisis, reflexión y sustento de dichas realidades discursivas construidas.

Poco importa para nuestro asunto indagar la génesis del pensamiento de la modernidad, sólo incitaría a entrar en un debate tautológico. Lo que es pertinente no olvidar es que cada época está estrechamente vinculada con la construcción teórica de sus realidades. En suma, basta con señalar, independientemente del planteamiento o la época a la que se haga alusión, que es innegable la constante de que sociologías y modernidad son discursos y gramáticas que se entrecruzan y confluyen siendo interdependientes y metacríticas a la vez.

No hay duda de que la lógica interdependiente entre modernidad y sociologías es conocida por Martuccelli, pues el análisis de textos y de autores parece reforzar dicha mirada. A decir del autor, la interdependencia entre modernidad y sociologías se encuentra en la voluntad por parte del análisis sociológico de construir representaciones globales de la realidad que permitan aprehenderla, posesionarla. Es decir, tomar conciencia del cambio constante de la realidad del mundo, de la ruptura con la estabilidad de la tradición que se genera con el proyecto de la modernidad. En suma, estamos refiriéndonos a la toma de conciencia de que existe la construcción de un mundo pretendido real que se valida a su vez con la constitución de una armadura intelectual. Se trata de crearle a la construcción de un mundo, un discurso, un léxico y una gramática que la legitime. Recordemos que hay un impacto y una relación estrecha entre el conocimiento científico y la historia, así nos refiramos a las tecnologías de guerra que influyen directamente en las estrategias políticas, o bien a las teorías producidas por la misma sociedad y que influyen en los sentidos de las acciones de los grupos e individuos.

Sin abusar del análisis de esa relación, cabe señalar que es precisamente a partir de la lógica ambivalente, la tensión y el conflicto que se generan en esta interdependencia, que se encuentran los elementos que guían la reflexión del libro y la selección de los autores y escuelas allí presentados. Efectivamente, se trata de un documento en el que se analiza no solamente el aporte de diferentes autores emblemáticos de la teoría social (14 en total), sino también su relación intrínseca con la construcción de 
problemáticas afines con el proyecto de la modernidad. Así, más que introducir al lector al análisis sociológico de escuelas y autores, se trata de reflexionar, con base en ellos, sobre la modernidad misma, como si se tratase de un itinerario reflexivo del siglo $\mathrm{xx}$, arrojando a la evidencia la constitución valórica de la misma modernidad.

Todo lo anterior se sustenta en la medida en que, por una parte, la noción de modernidad denota un 'espiritu del tiempo' cuya base y fundamento es la constante contradicción y renovación con su propio presente. El proyecto de la modernidad ha engendrado los fenómenos de la implosión, de la autodestrucción y de la autocrítica, que son fácilmente visibles con la llamada racionalización (una de las características innegables del pensamiento de la modernidad). Toda la dinámica de la modernidad se puede encontrar en la voluntad, primero, de reemplazar el esoterismo del politeísmo de los valores por la imposición de una sola divinidad (teodicea), luego con la pretensión de privatizar la evanescencia de un único Dios (secularismo), pasando por la muerte del mismo ser humano (nihilismo), hasta llegar a la reforma y reestructuración de la sociedad misma (posmodernidad). Esto ha traído como resultado la constante de la diferenciación social entre un mundo y sus ideas, entre grupos de pertenencia y fragmentaciones grupales, entre lo tradicional y lo nuevo. Es como si el sentido de existencia del proyecto de la modernidad se sustentara en la renovación constante de su dinámica, aunque ello signifique la renovación de sus mismos principios y la exacerbación de autocrítica de su mismo proyecto. En suma, sociologías y modernidad no representan más que la relación estrecha entre realidades construidas y herramientas de análisis que las sustentan.

Por otro lado, el vocablo sociología contiene la intensa preocupación de entender, comprender, descifrar, solucionar esa contradicción-renovación planteada en el proyecto de la modernidad. En otras palabras, las sociologías (en su sentido epocal) son retributivas de las relaciones de tensión que se viven en la modernidad. La condición moderna de la existencia se ve acompañada de sus legitimaciones teóricas y epistemológicas.

Efectivamente, lo que el texto nos hace ver es que para cada condición valórica inscrita en la modernidad y para cada problemática influida por la realidad hay una teoría, una escuela o un pensador representativo. Lo anterior es un suceso que se repite además de manera constante y concomitante a lo largo del tiem- 
po, y que el libro que aquí reseñamos magistralmente destaca. Es por medio de los aportes teóricos, de las construcciones conceptuales de cada escuela, que se puede analizar la llamada modernidad. Se trata de una crítica desde la sociología del conocimiento que hace de los teóricos mismos los testimonios de los elementos contenidos en el proyecto de la modernidad.

Son tres los elementos centrales constitutivos de la dinámica de esta modernidad, y que fungen como ordenadores del texto y como líneas de reflexión del libro; a saber: la racionalización, la diferenciación social y la condición social de la cotidianidad.

Cada uno de estos elementos ha encontrado vida en teorías y escuelas durante más de tres generaciones de teóricos sociales y que representan las principales etapas en la generación de la teoría social durante más de cien años de existencia. Estas generaciones se pueden caracterizar como sigue: la generación de los fundadores o clásicos de la sociología, seguida por la de los sociólogos modernos o industriales, después por la de los llamados sociólogos contemporáneos o postindustriales, y finalmente, la de los sociólogos del mundo actual y de la globalización.

Así, para representar la reflexión en torno a la diferenciación social, el autor refiere a autores como E. Durkheim, Talcott Parsons, Pierre Bourdieu y Niklas Luhmann, que dan cuenta de la manera como las diferentes teorías sociológicas han ido a la par con la conformación normativa y moral de la sociedad.

En el mismo eje de análisis, Danilo Martuccelli estudia a autores como Max Weber, Norbet Elias, Herbert Marcuse, Michel Foucault y Jürgen Habermas para dar cuenta de la dinámica de la racionalización, concepto básico en la fundamentación del proyecto de la modernidad en las sociedades occidentales, y que ha atravesado a la vez la historia misma de las sociologías.

Finalmente, para hablar de la condición moderna de la sociedad, el autor refiere a la escuela de Chicago y evoca a autores como Georg Simmel, Irving Goffman, Alain Touraine y Anthony Giddens, quienes han dado voz en sus planteamientos reflexivos a las regularidades cotidianas que se han conformado con la instauración de la lógica de la modernidad; es decir, regularidades que se han constituido en el proceso de la racionalización y la diferenciación social. Así, la organización tanto de los autores como de las problemáticas evocados no es cronológica de acuerdo con el tiempo gregoriano, y mucho menos responde a alguna otra propuesta temporal surgida de la historia en tanto que disciplina; responde a una lógica más entrañable al entrecruzamiento 
y concatenación del tiempo. Una organización en espiral, si así se puede decir...

Entonces, para cada problemática social existe una representación de teóricos y escuelas específicos. "Sociologías" invocaría, así, no solamente la pertenencia a una escuela de pensamiento o la nominación de un sociólogo en particular, sino la imbricación de todas estas problemáticas en un análisis integral y global de la realidad social. Es evidente, entonces, que en un proceso donde se exacerba la racionalización de la vida, se genera una diferenciación social de envergadura que influye de una u otra manera en la condición social de la vida cotidiana de las sociedades, de los grupos y de los individuos.

De esta manera, organizar la reflexión en torno a la teoría social (sociologías) que se ha generado en ciertas escuelas sociológicas a lo largo de la historia de la disciplina, significa de igual manera establecer el estudio de lo que ha sido el llamado proyecto de la modernidad a partir de las problemáticas que se entrecruzan y se concatenan. En suma, se trata de un estudio que nos permite aprehender nuestro tiempo y nuestra memoria del mundo vivido por medio de las problemáticas que algunas escuelas han destacado, y no por medio del protagonismo de un autor o la esquematización del conocimiento sociológico en tiempo lineal.

En esa organización reflexiva del libro, nos encontramos con una propuesta que se aleja profundamente de tantos otros manuales de sociología que surgieron poco después del mismo nacimiento y legitimación de la disciplina, sobre todo a partir de los años cincuenta; verbigracia, el emblemático libro de sociología de Ritzer, o incluso el mismo libro de Jeffrey Alexander. Se podría decir que la propuesta de Martuccelli actualiza la voz que tendría el clásico libro de Nisbet sobre la Tradición sociológica.

Lo anterior nos llevaría, al menos por lo que podríamos entrever en la lectura del texto, a responder al planteamiento inicial de que no habría sociologías sin modernidad y viceversa. Sin duda, cada autor o escuela, organizados esquemáticamente en el libro, representa una problemática que se ve atravesada con la reflexión de otra problemática que corresponde a otra escuela y a otra época o generación de sociólogos. Este aspecto no sólo nos invita a entender la dinámica misma de la modernidad, sino que también nos permite dar cuenta de la continuidad de las diferentes reflexiones dominantes durante todo el siglo Xx. De esta manera, se puede observar que la anomia o la patología de la modernidad ha constituido una obsesión no sólo de la sociología 
durkheimiana, sino que también lo ha sido de otras escuelas o autores, como la escuela parsoniana, donde la integración perfecta y la coordinación de las acciones constituyen el otro lado de la moneda, o, si se puede decir, el otro lado de la obsesión teórica que se complementa con la de su antecesor. Incluso las lecturas de la dominación o la noción del habitus, caras a Pierre Bourdieu, dan cuenta de la misma obsesión societal. Todo ello sin olvidar, evidentemente, la influencia que un Parsons ejerció en la problematización de la contingencia a través de la diferenciación o los sistemas autorreferenciales de Niklas Luhmann.

Lo mismo sucede cuando hablamos de la jaula de hierro, el retorno y la pérdida de sentido y la guerra de los dioses entrañables a la sociología weberiana, y que se ven complementadas con la lectura de un Norbet Elias y su proceso de civilización como racionalización, o bien con la lectura de un Herbert Marcuse y su conocida racionalización unidimensional, o incluso cuando nos referimos a la racionalización como sujetamiento de los individuos característica de los análisis foucaultianos. Dato curioso de encontrar un puente entre el problema de la racionalización y el de la diferenciación social en las reflexiones habermasianas alrededor de la ruptura de la concepción unitaria del mundo y las promesas de la modernidad.

Finalmente, cabe elogiar el protagonismo dado en el texto a Georg Simmel para emblematizar la reflexión en torno a las formas sociales, la vida mediada y la tragedia de la cultura, que se ven auspiciadas en un segundo tiempo con las reflexiones sobre la ciudad y la modernidad, la desorganización social y el hombre marginal de la Escuela de Chicago. Más aún, la reflexión alrededor de la condición moderna se ve consolidada con los aportes de las realidades múltiple, frágil y furtiva de un Erving Goffman, las problemáticas de la sociedad programada, el retorno del sujeto y el relato de la modernidad expuestas por Alain Touraine, o bien con las indagaciones de la teoría de la estructuración, la llamada modernidad tardía y la reflexividad y auto-identidad de Anthony Giddens.

Todas estas secuencias no hacen más que corroborar que enfoques y preocupaciones teóricos particulares sobre la modernidad han podido ser transmitidos a lo largo de la historia de la disciplina y que todos ellos forman, parafraseando al sociólogo de lo cotidiano, tribus que como tales son cómplices de sus mismas obsesiones teóricas. 
La cuestión de fondo de todo esto viene precisamente con el plural de sociología, y no se trata de puntillismo maniático de número, ni tampoco, creo, de una tendencia políticamente correcta, en tiempos que así se exige, por parte del autor al empecinarse en hablar en plural. Más bien se trata de toda una lógica inscrita en la construcción de sentido de una época que ha permeado todas las dinámicas societales a lo largo del siglo xx. No es casual tampoco que haya una breve mención de las llamadas sociologías de la posmodernidad. Allí una vez más la pertinencia de retomar la cuestión de si habría modernidad sin sociologías... Es precisamente porque la modernidad puede tener diversas interpretaciones, diversos análisis, diversos enfoques, que era necesario hacer un trabajo de reflexión al respecto. No obstante, parece claro que las sociologías a las que se hace alusión en el texto corresponden, ciertamente, a una diversidad de enfoques, pero bajo el esquema de una modernidad específica. Quizá la clave de lectura del libro mismo tenga que ver con el hecho de que se hace alusión a una modernidad concreta, a la modernidad occidental instrumental y sus corolarios, como son las sociologías de corte explicativo (exceptuando la de Weber, Simmel y Goffman).

Por lo pronto, limitémonos a dialogar con lo establecido y que parece más evidente en el libro aquí reseñado; a saber: la dependencia de ciertas sociologías respecto a la dinámica que representa la modernidad en sí.

Existen varios aspectos, mencionados en el texto, que hacen que la modernidad sea inseparable del nacimiento formal dominante de la sociología:

a) Ella da cuenta de una sociedad contemporánea; es decir, de una sociedad que vive en un tiempo presente y que se proyecta en un futuro, conteniendo por lo tanto un cuestionamiento en relación con su pasado. El presente es la esencia de lo vivencial, sin dejar de perder su dependencia de los referentes del pasado, que le permiten asumir un futuro particular.

b) La modernidad da cuenta principalmente de las incertidumbres que se generan con la dinámica de lo vivencial y el constante cuestionamiento a formas establecidas, de manera que la sociología se ve forzada a crear en cada momento sentido a las prácticas sociales, mismas que se ven atravesadas por esa tradición de lo nuevo inscrito en la lógica de la modernidad. La cuestión, en todo ello, es que siempre quedan res- 
quicios de incertidumbre que las sociologías no llegan a borrar, lo que explica la continuidad de escuelas y problemáticas en la conformación de la teoría social.

c) La modernidad proviene de este doble movimiento de construcción de representaciones globales y la conciencia inmediata de esta distancia con la realidad. Se trata de la conciencia que se genera entre la distancia simbólica que hay entre el pasado y el presente, entre la tradición y la renovación.

Por tanto, es necesario tener en mente tres fases principales que fundamentan el relato sociológico de la modernidad:

a) La distancia resentida por el actor entre sus expectativas y la realidad.

b) El resentimiento y la toma de conciencia de encontrar dinámicas contrarias a sus costumbres.

c) La necesidad de ordenar esta vivencia, al tiempo de conformar una estructura interpretativa que haga menos extraña su comprensión.

Esto significa que las sociologías de la modernidad nacen de la conciencia de la distancia que hay entre el presente y el pasado y de la tensión que hay con esta extrañeza de la experiencia.

Por ello, el autor se pregunta hasta qué punto la sociología es inseparable de una toma de conciencia histórica del sentimiento de ruptura con el pasado. El vocablo ruptura toma aquí toda su importancia en la dependencia de la sociología con la modernidad, pues ella da cuenta de la continuidad de la tradición de lo nuevo que se da en la dinámica propia de la modernidad, y de la necesidad cada vez mayor de dar sentido a cada modernidad que se inventa y reinventa hasta oponerse a ella misma. De ahí surge la exigencia de analizar cada época interna en el proceso mismo de la modernidad. Esto es lo que significa analizar, a partir de la sociología del conocimiento, o a partir de la meta-sociología, los aportes de la teoría social y su conexión con el "espíritu del tiempo" que los envuelve.

Parece entonces que la lectura de la modernidad propuesta en el texto se enfoca principalmente en una visión de desgarre, de fragmentación, de constantes jaloneos entre los actores y el sistema. Esto deja entrever una visión teleológica que, sin duda, es el reflejo de la modernidad dominante en el siglo xx y de las 
miradas evocadas en la teoría social. Ahora bien, es precisamente esta visión, asociada con los autores y las escuelas esbozados, lo que amerita una detenida lectura del texto, pues esclarece muy de cerca la relación del proyecto de la modernidad con la producción teórica.

Visto de esta manera, el libro presenta un enfoque de la modernidad de corte dialéctico más que integrativo, pues hay un empecinamiento por parte del autor en dar sentido y describir estas incertidumbres, estas rupturas, esta ambivalencia de la tradición a lo nuevo, y se olvida o se distancia, en el mejor de los casos, de una mirada de conjunto donde las rupturas y resquebrajamientos no serían más que parte de la dinámica societal que se ha vivido con el proyecto de la modernidad, y donde los procesos de lo imaginario, los sentidos de pertenencia, las emociones vividas en la cotidianidad, son los que subrepticiamente han conformado lo social. La evocación de autores como Simmel alude a estas dinámicas, que sin embargo se pierden frente a la vastedad de los demás autores que han fijado su mirada más en la visión de fragmentación, dualidad o dicotomía de la realidad social.

\section{El paradigma de la modernidad}

Esta problemática, definida así, nos permite cuestionar si la noción de modernidad no haría referencia a la noción de paradigma (en términos de Khun), que no es otra cosa que la capacidad de un término de convocar a un grupo de estudiosos a debatir y reflexionar al respecto. Se trataría de paradigmas en el interior mismo de lo que dio sustento al advenimiento de la sociología, pues, como se puede ver en el libro, no hay duda de que la reflexión en torno a la modernidad ha invocado a diferentes escuelas y posicionamientos investigativos que, confluyendo en una misma temática desde diferentes enfoques, han dado vida por más de cien años a la disciplina.

\section{Gramáticas de la modernidad}

Hay dos lecturas que se complementan en el libro y que nos dan pauta para decir que se trata de una propuesta bastante innovadora para hablar de dos temas: modernidad y sociología, desde dos miradas: pedagógica y heurística. Las dos lecturas podrían ser clasificadas como técnicas y críticas. 
Aspectos técnicos:

1. De manera general y un poco más en la superficie, se puede decir que el libro podría ser efectivamente un pretexto para hablar, a partir de diferentes autores, sobre el tema que le atañe al autor: la modernidad; es decir, un tipo específico de modernidad. Esta modernidad es claramente la que Weber señala en la introducción de su polémica obra sobre el espíritu del capitalismo y la ética protestante; es decir, la modernidad que promueve la racionalidad instrumental y la constante inversión en la construcción de empresas y proyectos nuevos.

2. En segundo lugar, se podría hablar, sin duda, de un libro de iniciación al panorama de la reflexión sociológica desde el fundamento mismo de la existencia de esa reflexión, lo cual si bien nunca va a reemplazar las fuentes originales, sí nos permite imbuirnos en un modo de leer la sociología.

3. También se trata de un diálogo entre sociólogos que evoca otras lecturas más allá de las que pudiéramos tener directamente de los teóricos de la sociología. Es una manera de sacarle más jugo a lo que se creía que ya no se podía exprimir. En otras palabras, darle vida a los clásicos y a los autores que se presentan como verdaderos constituidores de escuelas de pensamiento.

Aspectos críticos:

Estos aspectos son los más significativos en la obra, pues son los que distinguen a esta propuesta de muchas otras, pero que por supuesto no está separada de los aspectos técnicos ya mencionados.

1. Se trata efectivamente de una lectura socio-histórica sobre la sociología, sobre el recorrido de la sociología en sí misma en el siglo que acaba de concluir. Esta lectura se inscribe en un análisis que se podría denominar meta-sociológico, o de lo que unos llaman la sociología del conocimiento. Esto significa que no se trata de una lectura que haga teoría per se, sino que habla de la construcción de la teoría en sí, dialoga a propósito de la teoría misma, lo cual ya representa una contribución mayor al mismo conocimiento sociológico.

2. Ahora bien, además de tratarse de una lectura crítica sobre la sociología, la elección de ciertos autores contribuye a visualizar la manera como ciertas miradas y temas han 
dominado en el ámbito de la producción investigativa. Esta combinación hace que la lectura ofrezca nuevas alternativas de acercamiento, otros enfoques tanto a los autores y a la sociología como a la noción misma de modernidad. Se trata por tanto de una lectura hermenéutica de la sociología; es decir, de una lectura re-actualizada, tomando en cuenta el momento, las coyunturas y los tiempos que se viven, no sólo de los autores en su tiempo, sino también de los autores que han hablado de los mismos autores en otros tiempos. En suma, no es lo mismo la lectura de Weber por parte de sus contemporáneos, la lectura que hizo Parsons de Weber o la que hizo Giddens de Weber a partir de Parsons. Éste es sin duda uno de los aspectos más relevantes del libro. Por tanto, la lectura que se hace de los autores escogidos refleja claramente las preocupaciones y concepciones de nuestro tiempo, que no es sino la fragmentación, la separación, el pluralismo, etc.

3. Finalmente, se trata de una lectura analítica de la modernidad en términos epistemológicos; es decir, de una deconstrucción sobre la manera como se ha ido constituyendo el análisis mismo de la modernidad.

Para llevar a cabo estas lecturas, el autor parte de lo que se conoce como el análisis matricial de la modernidad, que es en cierta medida la manera como se puede comprender la continuidad de la reflexión sociológica y al mismo tiempo subrayar el rol activo que esta intuición inicial tiene en cada uno de los autores cuando interpretan la modernidad de su tiempo e intentan responder a los cambios históricos y sociales de su época. La matriz como clave de análisis permite estructurar las múltiples maneras de escribir la historia de la sociología y de sus divisiones. Nos permite ver cuál es la visión de la modernidad que está en juego en la obra de cada autor seleccionado, y también, cuáles son los enfoques políticos que están en juego en cada época. Cuáles son pues los contornos y las inflexiones de estas matrices.

Finalmente, la idea de matriz insiste sobre la profunda continuidad de la mirada sociológica a lo largo del siglo xx e intenta dar cuenta de los procesos históricos en los que está imbuida cada modernidad, lo que lleva a generar un arsenal imaginativo mayor en la teoría sociológica que permita dar cuenta de dichos procesos.

En otras palabras, la utilización de la matriz como herramienta de lectura de la sociología y la modernidad, nos permite si- 
tuarnos en un enfoque intermitente, en un nivel meso entre los puntos comunes y las diferencias de los autores y escuelas con su coyuntura histórico-geográfica; verbigracia, el análisis del individuo que realiza cada versión sociológica.

Hay dos aspectos a tomar en cuenta antes de concluir con este punto: primero, el hecho de que se hable de matriz, y no de matrices. Efectivamente, al hablar en términos de matrices nos vemos forzados a hablar de una diversidad de posibles aspectos que permitan describir la dinámica observada, o, en todo caso, reflejar las posibles preocupaciones sociológicas que se puedan esbozar en torno a la dinámica de la modernidad. Precisamente, si algo se subraya de la historia de la sociología, y es aquí el punto nodal del porqué se habla de una sola modernidad y no de modernidades, es que la disciplina precisamente se ha dividido en diferentes matrices analíticas, hecho que ha generado que se presuponga en la actualidad la existencia de fragmentaciones y querellas entre corrientes sociológicas, sobre todo en torno a la manera como se deben estudiar los fenómenos sociales. No entraremos mucho en debate al respecto, pues nos saldríamos del tema específico del libro; sólo basta con decir que al menos dos tipos de modernidades se han gestado, asociadas con dos escuelas de pensamiento específicas, y que el autor privilegia una de ellas. Nos referimos a la modernidad vacía, heredera de un platonicismo cristiano donde la realidad se pretende controlar, manipular y explicar, y a la modernidad plena, vertiente de un aristotelismo cuya meta es comprender un mundo complejo y vasto. Es evidente que la modernidad que se favorece en el análisis del texto es aquella influida por formas explicativas de ver el mundo social. De ahí la elección de los autores y de las miradas teóricas.

Se pudo haber pensado en otros autores y escuelas para reflexionar en torno a la modernidad y a la producción de teoría social. Autores que irían desde Dilthey, Husserl, Alfred Schütz, Bachelard, Bergson, Gabriel Tarde, Garfinkel, Balandier, Edgar Morin, Gilbert Durand, Pierce, Mead, Wright Mills, entre tanto otros, pero la elección de estos autores hubiera llevado a hablar de otras modernidades y de otras sociologías que sin duda no han predominado en el mundo de las ideas sociológicas, aunque han acompañado a la historia sociológica dominante. Ahora bien, cabe insistir en el acierto del autor de reflexionar sobre las propuestas de Simmel, la Escuela de Chicago y Goffman, que sin duda han sobresalido de la propuesta explicativa de las demás escuelas y autores, y que defienden y se acercan más al otro tipo 
de modernidad a la que nos referíamos. Es un acierto en la medida en que en la actualidad, las problemáticas dominantes tienden a rescatar o a hacer un llamado a estas otras propuestas interpretativas. Sólo nos queda señalar que faltaría seguir con los planteamientos del autor complementándolos con otra obra que incluyera a dichos autores.

Ahora bien, para concluir con la reflexión sobre la matriz como herramienta de análisis para leer la complejidad de la realidad social, es necesario mencionar que los ejes ordenadores insertos en la propuesta del autor son el orden y la libertad. En efecto, se trata de dos ejes analíticos que se contradicen, se contraponen pero se complementan. Si hay algo que caracteriza a todas las propuestas teóricas, y a la preocupación de todos los autores seleccionados en este libro, es la obsesión por encontrar un orden en un espacio de libertad. No hay nada más claro para describir efectivamente los supuestos valóricos sobre los que descansa la modernidad dominante (con todo y sus contenidos: diferenciación social, racionalización y condición moderna) que estos dos ejes investigativos. Sin duda encontraremos aquí, de igual manera, los presupuestos de un pensamiento que consolidaron el cristianismo reformado, tal y como Weber hace cien años lo manifestó con el ascetismo intra-mundano calvinista.

La matriz que guía el resto de la lectura a partir de los ejes de investigación: orden y libertad, es la tríada antes mencionada de la diferenciación social, la racionalización y la condición social.

En relación con la diferenciación social, el autor plantea que no ha habido otro elemento de la matriz de la modernidad que haya marcado tanto la reflexión sociológica, al punto que ésta es siempre solicitada en las interpretaciones dadas al proceso de modernización, cualquiera que sea el autor de referencia. Se trata de la diferenciación que va de lo homogéneo a lo heterogéneo, la diferenciación que se encuentra en los procesos de la división del trabajo y de la singularidad que se acrecienta con este proceso. La diferenciación a la que se hace alusión tiene que ver con la diversificación de grupos, de roles, de normas posibles, que plantea la problemática de la significación de patrones culturales y de principios funcionales de integración social. Tiene que ver con el aspecto de la diferencia y la integración, de la norma y la anomia entre el equilibrio inestable y la integración moral, entro lo contingente y la normatividad; aquí se plantea el paso de la reproducción social y de la inadaptación cultural de los individuos que cargan una historia personal y colectiva. Finalmente, 
este elemento de la matriz tiene que ver con la cuestión de si la diferenciación social no es factor del funcionamiento mismo de la sociedad.

En lo que concierne al elemento matricial de la racionalización, el autor plantea que siendo uno de los grandes elementos de la matriz de la modernidad, es igualmente uno de los más ambiguos y equívocos. De ahí la necesidad de distinguir la racionalidad de Occidente como forma diferenciada de actuar en el mundo, junto con su corolario, que tiene que ver con el estatus de las instituciones modernas, en comparación con otras formas racionales existentes en el mundo. Pero también tiene que ver con las posibilidades de emancipación, sea que resulten en factores constructivos para la especie humana, sea que se trate de factores destructivos para la civilización o bien sea finalmente que se refiera a la unidimensionalidad de la racionalidad y de su ineluctable permanencia. Para unos autores, la racionalización es el factor de expansión de las sociedades, para otros es el factor de disciplinamiento de los grupos y los individuos, para otros finalmente es la única opción de comunicación entre los pueblos.

Finalmente, en lo que concierne la condición moderna, el autor destaca que se trata de un elemento de la matriz poco explorado anteriormente, de ahí que se señale que se trata de un tipo de modernidad sociológica distinto al de tipo explicativo. Esta mirada esta más allá de los juicios morales entre lo conveniente o no para una sociedad, y más bien destaca la organización de la sociedad alrededor de las paradojas y las contradicciones de la vida moderna. Aquí se toma conciencia de otros factores en los grupos humanos que construyen sociedad, como, por ejemplo, el aspecto fugitivo, errante y eterno de lo vivencial. Se trata de un análisis de lo transitorio, de lo contingente, de la manifestación fenomenológica de la existencia, de la situación metafísica del ser humano, de la importancia de la dimensión espacial en los grupos humanos. Así, se habla de la astucia, de la socialización, de la fragmentación de la experiencia como formas de conocimiento de lo social. De esta manera, no se trata de una perspectiva radicalista donde cada individuo se vuelve el amo de su destino y de lo que le rodea y de sus propias significaciones, sino que resalta lo fragmentado del individuo a partir de estas disyuntivas. Ahora bien, la mirada aquí es que finalmente la modernidad es gozosa de sí misma, con una formidable explosión de horizontes posibles y abiertos, que están ahí para ser analizados, 
sin más objeto que su comprensión en el presente en el que se observa y se vive.

\section{¿Modernidad o modernidades?}

Sólo nos queda agregar como frase conclusiva, que el texto aquí reseñado, además de fungir como recorrido reflexivo de la sociología y de la modernidad, nos da pauta a otros debates no explorados anteriormente, como el referido al lugar que retoman en la actualidad otras sociologías pertenecientes a otras modernidades, ya no digamos aquellas que se ubican fuera del contexto occidental, sino oriundas del mismo contexto en el que se gestó el proyecto prometeico de la modernidad y que ha encontrado hoy formas de desgaste. Sin duda esto nos lleva a considerar el planteamiento en términos de modernidades; es decir, en términos de la existencia de una pluralidad de formas de relacionarse con el alrededor, y de la pluralidad de formas de analizar la misma modernidad; ya no quizá con la mirada de la ruptura y la fragmentación, sino con la de la oposición complementaria que tienen los contrarios en la conformación de lo social en la vida cotidiana. Esto nos induce finalmente a corroborar que para un tipo de acercamiento reflexivo a nuestro tiempo, a un tipo específico de modernidad, existe una referencia a un tipo específico de sociología. Éste podría ser precisamente el hilo de Ariadna del documento aquí presentado, pero también de la reflexión del mundo que vivimos y de las herramientas de conocimiento que le adjudicamos.

A partir de estas nuevas exploraciones sobre la modernidad, es importante dar cuenta de la importancia del libro reseñado y de su necesaria traducción en un futuro al castellano. Esto no hará más que enriquecer nuestros enfoques y acercamientos teóricos en el mundo hispano.

Daniel Gutiérrez Martínez

El Colegio Mexiquense, A.C. Correo-e: dgutierrez@cmq.edu.mx.

Daniel Gutiérrez Martínez es candidato a doctor en ciencias sociales con especialidad en sociología por el Centro de Estudios Sociológicos de El Colegio de México, A.C., maestro en antropología del desarrollo por el Instituto de Estudios del Desarrollo Económico y Social (IEDES), París I Panthéon-Sorbonne, y licenciado en 
sociología económica por la Universidad de París I Panthéon-Sorbonne. Es profesor-investigador asociado en la Facultad de Ciencias Políticas y Sociales de la Universidad Nacional Autónoma de México e investigador asociado en el Programa Interdisciplinario de Estudios sobre las Religiones (PIER) en El Colegio Mexiquense, A.C. Ha colaborado en diversos suplementos culturales de periódicos mexicanos como La Crónica y Unomásuno, y en revistas especializadas como Estudios Sociológicos, de El Colegio de México. Su más reciente libro: Multiculturalismo: perspectivas y desafíos, Siglo XXI Editores-Posgrado, unAM-El Colegio de México, A.c. 